\title{
ORIGINAL
}

\section{Nasal high-flow preoxygenation for endotracheal intubation in the critically ill patient: a randomized clinical trial}

\author{
Christophe Guitton ${ }^{1 *}$, Stephan Ehrmann ${ }^{2}$, Christelle Volteau ${ }^{3}$, Gwenhael Colin ${ }^{4}$, Adel Maamar ${ }^{5}$, \\ Vanessa Jean-Michel ${ }^{6}$, Pierre-Joachim Mahe ${ }^{7}$, Mickael Landais ${ }^{1}$, Noelle Brule $^{8}$, Cedric Bretonnière ${ }^{8}$, \\ Olivier Zambon ${ }^{8}$ and Mickael Vourc' $h^{8}$
}

(c) 2019 Springer-Verlag GmbH Germany, part of Springer Nature

\begin{abstract}
Purpose: Preoxygenation with high-flow therapy by nasal cannulae (HFNC) is now widespread in the intensive care unit (ICU). However, no large randomized study has assessed its relevance in non-severely hypoxemic patients. In a randomized controlled trial (PROTRACH study), we aimed to evaluate preoxygenation with HFNC vs. standard bagvalve mask oxygenation (SMO) in non-severely hypoxemic patients during rapid sequence intubation (RSI) in the ICU.

Methods: Randomized controlled trial including non-severely hypoxemic patients requiring intubation in the ICU. Patients received preoxygenation by HFNC or SMO during RSI. HFNC was maintained throughout the intubation procedure whereas SMO was removed to perform laryngoscopy. The primary outcome was the lowest pulse oximetry $\left(\mathrm{SpO}_{2}\right)$ throughout the intubation procedure. Secondary outcomes included drop in $\mathrm{SpO}_{2}$, adverse events related to intubation, and outcome in the ICU.

Results: A total of 192 patients were randomized. In the intent-to-treat analysis, 184 patients (HFNC $n=95 ;$ SMO $n=89$ ), the median [IQR] lowest $\mathrm{SpO}_{2}$ was $100 \%[97 ; 100]$ for HFNC and 99\% $[95 ; 100]$ for the SMO group $(P=0.30)$. Mild desaturation below 95\% was more frequent with SMO (23\%) than with HFNC (12\%) (RR 0.51, 95\% Cl 0.26-0.99, $P=0.045)$. There were fewer adverse events in the HFNC group (6\%) than in the SMO group (19\%) (RR 0.31, 95\% Cl $0.13-0.76, P=0.007$ ), including fewer severe adverse events, respectively $6(6 \%)$ and $14(16 \%)$ with HFNC and SMO (RR $0.38,95 \% \mathrm{Cl} 0.15-0.95, P=0.03)$.

Conclusions: Compared with $\mathrm{SMO}$, preoxygenation with HFNC in the ICU did not improve the lowest $\mathrm{SpO}_{2}$ during intubation in the non-severely hypoxemic patients but led to a reduction in intubation-related adverse events.

Trial registration: Clinical trial Submission: 7 March 2016. Registry name: Benefits of high-flow nasal cannulae oxygen for preoxygenation during intubation in non-severely hypoxemic patients: the PROTRACH study. Clinicaltrials.gov identifier: NCT02700321. Eudra CT: 2015-A00145-44. CPP: 15/13-975 (Comité de protection des personnes de Rennes). URL registry: https://clinicaltrials.gov/ct2/show/record/NCT02700321.
\end{abstract}

\footnotetext{
*Correspondence: cguitton@ch-lemans.fr

1 Service de Réanimation Médico Chirurgicale, USC, Centre Hospitalier du Mans, 194 Avenue Rubillard, 72037 Le Mans Cedex 9, France

Full author information is available at the end of the article 
Keywords: Preoxygenation, High-flow oxygen therapy by nasal cannulae, Intubation, Apneic oxygenation, Intensive care unit

\section{Introduction}

Endotracheal intubation is a procedure that is performed daily in the intensive care units (ICU). Nevertheless, it remains a life-threatening event and causes more than $25 \%$ of severe complications such as oxygen desaturation, hemodynamic instability, and death [1-3], driving intensive research efforts in the field [4-6].

Preoxygenation is a key component of intubation in order to stock up oxygen in functional residual capacity (FRC) volume, representing the main oxygen reservoir during apnea [7]. After the induction of general anesthesia, it extends the duration of apnea until hypoxemia occurs, i.e., oxygen desaturation below a critical pulse oximetry $\left(\mathrm{SpO}_{2}\right)$ value [8]. In critically ill patients, several pathological conditions compromise optimal preoxygenation such as high peripheral oxygen uptake, hemodynamic instability, or altered consciousness. In this setting, preoxygenation with the standard bag-valve mask oxygenation (SMO) followed by rapid sequence intubation (RSI) is proposed [9] to reduce the risk of desaturation and aspiration. RSI includes immediate intubation after hypnotic administration without manual face mask ventilation to minimize the time from induction to intubation. SMO is removed during laryngoscopy-meaning that oxygenation of the patient is interrupted. Alternatives such as high-flow therapy by nasal cannulae (HFNC) could prove beneficial. Indeed, considering the encouraging results in patients presenting acute hypoxemic respiratory failure [10], HFNC has been proposed as a preoxygenation device for intubation in the ICU. This device can deliver up to $60 \mathrm{~L} / \mathrm{min}$ with an inspired fraction of oxygen of up to $100 \%$ [11], generate a moderate positive supraglottic end-expiratory pressure, and reduce the patient effort [12]. Contrary to SMO, HFNC makes it possible to hold nasal prongs in place after induction of general anesthesia and during laryngoscopy, to perform apneic oxygenation throughout the intubation procedure [13]. Furthermore, tolerance of HFNC has repeatedly been shown to be excellent whereas SMO can cause discomfort and claustrophobia since the mask is tightly applied to the patient [14]. HFNC is a widespread device in the ICU but there is no large randomized study that has evaluated its benefits as a preoxygenation device for intubation in non-severely hypoxemic patients. We recently reported that this device failed to prevent desaturation during intubation in severely hypoxemic patients [3]. In non-severely hypoxemic patients, a recent

\section{Take-home message}

This is the first randomized multicenter study comparing the use of HFNC with SMO for the preoxygenation of non-severely hypoxemic patients in the ICU. In this setting, compared to SMO, preoxygenation with HFNC did not improve the lowest $\mathrm{SpO}_{2}$ during intubation but led to a reduction in intubation-related adverse events.

before-after study suggested that HFNC could improve preoxygenation and reduce severe hypoxemia during intubation [15].

The main objective was to determine the efficacy of HFNC for preoxygenation compared with SMO as measured by the lowest level of pulse oximetry during RSI recorded through $\mathrm{SpO}_{2}$. Secondary objectives were to compare intubation procedures, related side effects, and patient outcome in the two groups.

\section{Methods}

\section{Study design, setting, and ethical considerations}

The PROTRACH study was a multicenter, randomized, controlled, open-label clinical trial. The main objective was to determine the efficacy of HFNC to prevent oxygen desaturation during intubation in the ICU compared with standard of care. Patients were enrolled from April 2016 to June 2017 in seven French ICUs (four medical, two medical-surgical, and one surgical) and followed for 28 days. An independent safety committee oversaw the trial.

Three methods of consent were available; patients were included after written informed consent whenever possible. Most patients were included after the written informed consent of their next-of-kin or following an emergency inclusion procedure. The appropriate ethics committee approved this study protocol in September 2015 (15/13-975).

\section{Participants}

Eligible patients were adults (18 years or older), requiring intubation in the ICU, without severe hypoxemia defined as a ratio of arterial partial pressure in oxygen to fraction of inspired oxygen $\left(\mathrm{PaO}_{2} / \mathrm{FiO}_{2}\right)$ below 200 within $4 \mathrm{~h}$ before inclusion. To determine the $\mathrm{PaO}_{2} / \mathrm{FiO}_{2}$ ratio on inclusion, the $\mathrm{FiO}_{2}$ specified by the manufacturer for Venturi mask or corresponding to the oxygen flow for standard nasal prongs [16] was considered.

Exclusion criteria were intubation without rapid sequence induction (i.e., in case of cardiac arrest), fiberoptic intubation, asphyxia requiring immediate 
intubation, any nasopharyngeal anatomical obstacle, grade 4 glottis exposure on the Cormack-Lehane scale documented in the medical history, adults subject to legal protection, pregnancy, lack of consent, patients who had already undergone intubation in the ICU (i.e., extubation failure) or who were already participating in an interventional study on preoxygenation.

\section{Randomization}

Randomization used fixed blocks of six patients (ratio 1:1) and was stratified by center. The study statistician generated the allocation list. Patients were allocated to one of the two preoxygenation strategies using a secure computer-generated online remote system controlled by the independent research promotion unit at the University Hospital of Nantes which had no role in patient recruitment.

\section{Intervention: preoxygenation and intubation procedure}

The study design is described in Supplement 1. Preoxygenation and intubation were performed according to international recommendations in all centers [9]. The intubation team was composed of one junior and one senior.

1. Preoxygenation was started according to the randomization group for a 4-min period [17] in a head-up position. In the intervention group, preoxygenation was performed with HFNC (AIRVO $2^{\mathrm{TM}}$; Fisher \& Paykel Healthcare, Auckland, NZ), nasal prongs set at $60 \mathrm{~L} / \mathrm{min}$ flow of heated and humidified pure oxygen (100\% fraction of inspired oxygen, $37{ }^{\circ} \mathrm{C}$ ). Large or medium nasal cannulae were chosen according to the patient's nostril size to limit air contamination. In the control group, preoxygenation was performed with SMO set at $15 \mathrm{~L} / \mathrm{min}$ oxygen flow with a disposable self-inflating resuscitator with a reservoir bag manually held in place ensuring air tightness.

2. At the end of preoxygenation, RSI including immediate intubation after administration of the hypnotic drugs was performed in all patients. Drugs and the use of a neuromuscular blocking agent were left to the discretion of the physician.

3. During laryngoscopy and intubation, HFNC was maintained in place throughout the procedure in an attempt to achieve apneic oxygenation. In the control group, SMO was removed in order to perform laryngoscopy.

4. In case of difficult intubation, the stylet was the first alternative device which was proposed [18] if external laryngeal manipulation did not improve glottis vision.
5. At the end of intubation, the correct position of the tracheal tube was confirmed by using capnography [19].

\section{Endpoints}

The primary endpoint was the median lowest $\mathrm{SpO}_{2}$ during intubation, from the beginning of the laryngoscopy until the patient was connected to the mechanical ventilator. To ensure exhaustive data collection, a research nurse and an external observer concurrently collected $\mathrm{SpO}_{2}$ fluctuations during the intubation.

Secondary endpoints were $\mathrm{SpO}_{2}$ from the beginning of preoxygenation to the end of intubation focusing on desaturation (below 95\%, 90\%, 80\%); the rate of difficult intubation [20] and the intubation difficulty scale (IDS) score [21]; the need to proceed to face mask ventilation to correct desaturation; adverse events a priori classified as severe (death, cardiac arrest, $\mathrm{SpO}_{2}<80 \%$, severe hypotension defined by systolic blood pressure $<80 \mathrm{mmHg}$ or vasopressor initiation or $30 \%$ dose increment) or moderate (ventricular or supraventricular arrhythmia requiring intervention, esophageal intubation, vomiting with aspiration of gastric content, dental injury); organ failure during the first 5 days (SOFA score); time on the ventilator; length of stay in ICU; occurrence of ventilator-associated pneumonia and mortality rate on day 28 (full study protocol appears in Supplement 2).

\section{Sample size}

On the basis of previous studies we hypothesized that HFNC would improve the mean lowest $\mathrm{SpO}_{2}$ value by $5 \%$ (from $88 \%$ in the control group to $93 \%$ in the HFNC group) with a standard deviation of $\mathrm{SpO}_{2}$ of $12 \%$ [15, $22]$. With $80 \%$ power, a $5 \%$ type I error (two-sided tests), and 5\% attrition rate, the required sample size was 192 patients.

\section{Statistical analysis}

Baseline characteristics were described as count and percentage for categorical variables and mean and standard deviation or median and interquartiles for quantitative variables when assumption of normality was not met. An intent-to-treat statistical analysis was conducted. Comparisons of primary and secondary outcomes were performed using the Mantel-Haenszel Chi-square tests (or two-tailed Fisher exact tests when appropriate) for qualitative data and the Van Elteren tests for quantitative data to account for stratification factor (center). For qualitative data, relative risk (with adjustment for center effects) and 95\% confidence interval were estimated with the MantelHaenszel method. Multivariable analysis of $\mathrm{SpO}_{2}<90 \%$, $\mathrm{SpO}_{2}<80 \%$, and the occurrence of adverse events were performed using logistic regression. The most clinically 
relevant variables (skills of the first operator, difficult intubation, $\mathrm{MACOCHA}$ score $\geq 3$, and $\mathrm{PaO}_{2} / \mathrm{FiO}_{2}$ ratio at baseline) or associated with the randomization group $(P$ values $<0.20)$ in univariate analysis (age and NYHA class) were introduced simultaneously into the models. Odds ratios and their $95 \%$ confidence intervals (CI) were estimated. All tests were two-tailed. $P$ values of less than 0.05 were considered significant. No data was missing for the primary outcome. For secondary outcomes, missing data were clearly stated in the tables (see also Supplement 3), and no method of imputation was used. SAS software version 9.4 (SAS Institute Inc.) was used.

\section{Results}

\section{Patients}

In the seven participating ICUs, 192 patients were randomized in the study between April 2016 and June 2017. Three patients withdrew their consent, two patients were discovered to be under guardianship protection, and three patients improved before the start of the study and were therefore not intubated. We considered 184 patients (95 in HFNC and 89 in SMO) in the intent-to-treat (ITT) and 183 patients in the per-protocol (PP) analyses (Fig. 1). Per-protocol analysis excluded the only patient with protocol deviation in the HFNC group who did not receive preoxygenation with HFNC because no device was available. This patient did not present any complication during intubation.

At baseline, patients were mainly intubation for a medical condition with a median [interquartile range, IQR] simplified acute physiology score 2 (SAPS II) of
$45[35 ; 60]$ in HFNC and 41 [32; 57] in SMO; median [IQR] $\mathrm{PaO}_{2} / \mathrm{FiO}_{2}$ was $318 \mathrm{mmHg}$ [242; 396] in HFNC and $375 \mathrm{mmHg}$ [276; 446] in SMO. In the two groups, intubation was mainly performed for neurologic reasons in comatose patients: $68 \%$ in HFNC and $75 \%$ in SMO (Table 1). In order to prevent hypotension, fluid loading was performed before anesthesia induction in 41 (43\%) patients in HFNC vs. 37 (42\%) in SMO. Airways, operators, and technical aspects of intubation are described in Table 2. Mean (standard deviation, SD) MACOCHA score to predict difficult intubation was 2.0 (1.6) in HFNC and 2.2 (2.0) in SMO [23]. There was no betweengroup difference in doses or nature of hypnotics (see Supplement 3). All intubations were performed with two operators including a senior and the first operators were mainly (84\%) juniors. In the HFNC group, cannula sizes were large for $45 \%$ and medium for $55 \%$. No patient was lost to follow-up (see Supplement 3).

\section{Primary outcome: lowest $\mathrm{SpO}_{2}$ during the intubation procedure}

In the intent-to-treat (ITT) analysis (Table 3, Fig. 2), there was no significant difference between the median [IQR] lowest $\mathrm{SpO}_{2}$ during intubation in the HFNC group compared with the SMO group, 100\% [97; 100] versus $99 \%[95 ; 100]$ respectively, $(P=0.30)$. In the per-protocol (PP) analysis (attrition rate 1\%), the lack of difference persisted $(P=0.26)$. Although only non-severely hypoxemic patients were included, the lowest $\mathrm{SpO}_{2}$ during intubation ranged from $69 \%$ to $100 \%$ in HFNC and from $43 \%$ to $100 \%$ in SMO.

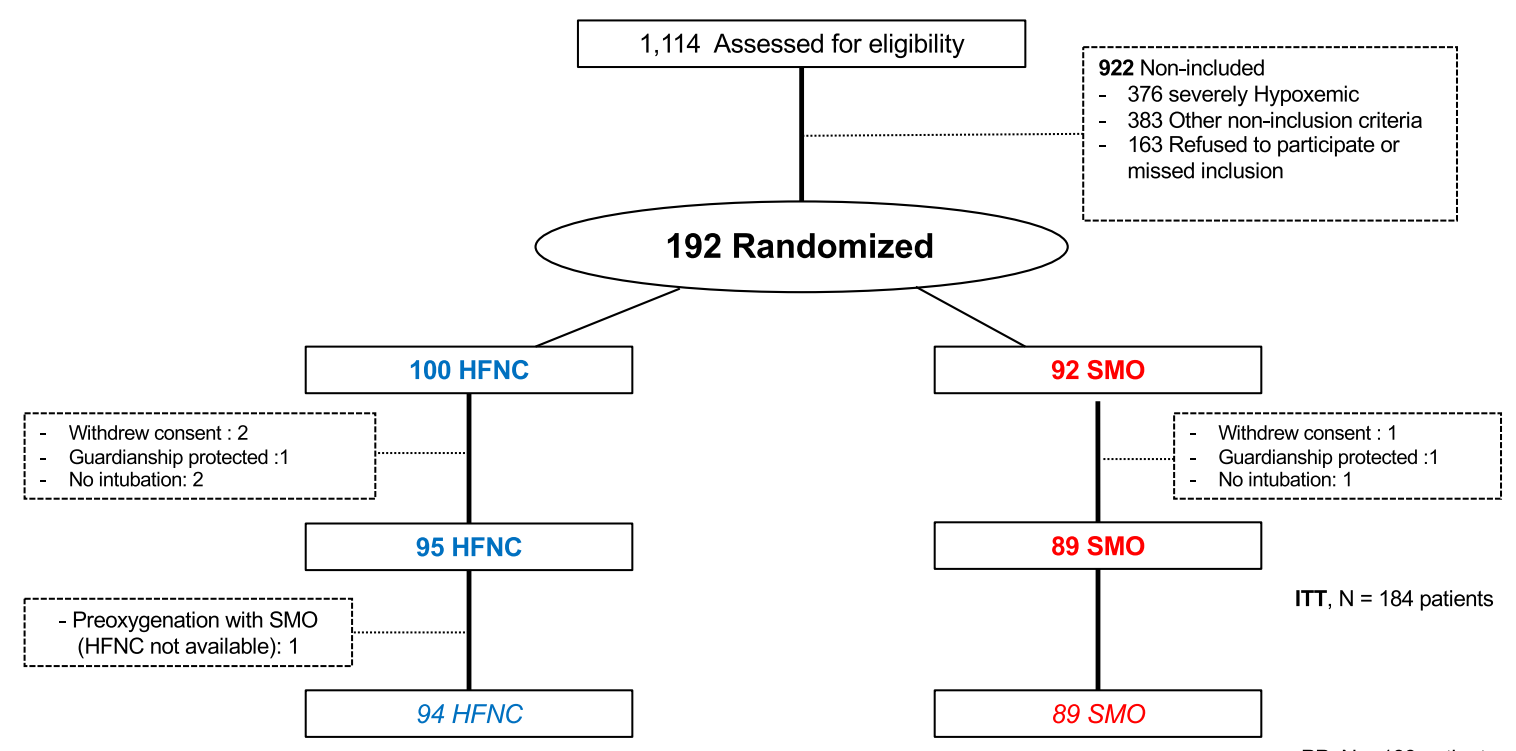

Fig. 1 Study flowchart. HFNC high-flow nasal cannulae, SMO standard bag-valve mask oxygenation 
Table 1 Baseline characteristics of the intent-to-treat population

\begin{tabular}{|c|c|c|}
\hline & $\begin{array}{l}\text { High-flow nasal cannulae } \\
n=95\end{array}$ & $\begin{array}{l}\text { Standard bag-valve } \\
\text { mask oxygenation } \\
n=89\end{array}$ \\
\hline Sex ratio M/F & $65 / 30$ & $62 / 27$ \\
\hline Age, median [IQR], year & $60[49 ; 72]$ & $61[41 ; 68]$ \\
\hline $\mathrm{BMI}$, mean $(\mathrm{SD})^{\mathrm{a}}$ & $27.0(6.6)$ & $25.9(5.5)$ \\
\hline Medical patients, $n(\%)$ & $90(95)$ & $87(98)$ \\
\hline SAPS II score, median [IQR] & $45[35 ; 60]$ & $41[32 ; 57]$ \\
\hline \multicolumn{3}{|l|}{ Comorbidities } \\
\hline Chronic heart failure (NYHA III or IV), n (\%) & 0 & $3(3)$ \\
\hline Chronic respiratory failure, $n(\%)$ & $8(8)$ & $10(11)$ \\
\hline Obstructive sleep apnea syndrome, $n(\%)$ & $1(1)$ & $5(6)$ \\
\hline COPD, $n(\%)^{b}$ & $8(9)$ & $8(9)$ \\
\hline Past upper airway tract cancer, $n(\%)$ & $1(1)$ & 0 \\
\hline Diabetes, $n(\%)$ & $11(12)$ & $8(9)$ \\
\hline Vasopressor support, $n(\%)$ & $11(12)$ & $12(14)$ \\
\hline Glasgow coma score, mean (SD) & $8[5 ; 14]$ & $9[6 ; 14]$ \\
\hline McCabe scale 2 or $3, n(\%)^{c}$ & $13(14)$ & $15(17)$ \\
\hline Functional status (class C or D KNAUS), $n(\%)^{d}$ & $8(8)$ & $14(16)$ \\
\hline \multicolumn{3}{|l|}{ Main indications for intubation, $n(\%)$} \\
\hline Acute respiratory failure & $14(15)$ & $7(9)$ \\
\hline Comatose patients & $65(68)$ & $67(75)$ \\
\hline Hemodynamic dysfunction & $7(7)$ & $4(4)$ \\
\hline Other & $9(10)$ & $11(12)$ \\
\hline \multicolumn{3}{|c|}{ Advanced oxygenation support before inclusion, $n(\%)$} \\
\hline Non-invasive ventilation & $6(6)$ & $8(9)$ \\
\hline HFNC & $3(3)$ & $2(2)$ \\
\hline \multicolumn{3}{|l|}{ Arterial blood gas oxygenation before intubation } \\
\hline $\mathrm{PaO}_{2} / \mathrm{FiO}_{2}$, median $[\mathrm{IQR}], \mathrm{mmHg}$ & $318[242 ; 396]$ & $375[276 ; 446]$ \\
\hline $\mathrm{PaCO}_{2}$, mean $(\mathrm{SD}), \mathrm{mmHg}$ & $36[30 ; 46]$ & $37[29.6 ; 47]$ \\
\hline $\mathrm{SpO}_{2}$, median $[\mathrm{IQR}], \%$ & $97[95 ; 99]$ & $98[96 ; 100]$ \\
\hline
\end{tabular}

$B M I$ body mass index, SAPSIl simplified acute physiological score (used to assess the severity of illness; range 0-194, with higher scores indicating higher risk of death), COPD chronic obstructive pulmonary disease, HFNC high-flow therapy by nasal cannulae

a Calculated as weight in kilograms divided by height in square meters

b COPD was considered if obstructive syndrome had been documented on functional explorations

c McCabe classification: cat 1, nonfatal disease; cat 2, ultimately fatal disease (within 4 years); cat 3, rapidly fatal disease

d KNAUS chronic health status: class A, normal health status; class B, moderate activity limitation; class C, severe activity limitation due to chronic disease; class D, bedridden patient

\section{Secondary outcomes}

All of the patients were successfully intubated and only one operator removed HFNC for laryngoscopic vision obstruction. At the end of the preoxygenation period, median [IQR] $\mathrm{SpO}_{2}$ was $100 \%$ [100; 100], $(P=0.66)$ in both groups (Table 3). During preoxygenation, jaw-thrust maneuver was performed in $13 \%$ of the patients in HFNC and in $33 \%$ in SMO (relative risk 0.38, 95\% CI 0.21-0.71, $P=0.0009)$. The median [IQR, min-max] length of intubation (namely, apnea duration) was higher in HFNC (1 [0.5; 1.9, 0-23]) vs. in SMO $(0.8[0.5 ; 1.4,0-18] \mathrm{min}$, $P=0.04$. Eleven patients (12\%) in HFNC vs. 20 (23\%) in
SMO experienced an $\mathrm{SpO}_{2}$ drop below 95\% (relative risk $0.51,95 \%$ CI $0.26-0.99, P=0.045)$. Six patients $(6 \%)$ in the HFNC group vs. $12(14 \%)$ in the SMO group experienced an $\mathrm{SpO}_{2}<90 \%(P=0.10)$. Two patients $(2 \%)$ in the HFNC group vs. 7 (8\%) in the SMO group experienced a severe $\mathrm{SpO}_{2}<80 \%(P=0.06)$. Overall, during intubation, 6 patients $(6 \%)$ in the HFNC group vs. 17 patients $(19 \%)$ in the SMO group (relative risk 0.31, 95\% CI 0.13-0.76, $P=0.007$ ) presented at least one intubation-related complication. Severe complications including $\mathrm{SpO}_{2}<80 \%$, severe hypotension, and cardiac arrest occurred in $6 \%$ of the patients in HFNC vs. $16 \%$ in SMO (relative risk 


\section{Table 2 Airway, preoxygenation, and intubation settings at baseline}

\begin{tabular}{|c|c|c|}
\hline & $\begin{array}{l}\text { High-flow nasal cannulae } \\
n=95\end{array}$ & $\begin{array}{l}\text { Standard bag-valve } \\
\text { mask oxygenation } \\
n=89\end{array}$ \\
\hline \multicolumn{3}{|l|}{ Airway description } \\
\hline At least two difficult mask ventilation criteria, $n(\%)^{a}$ & $46(48)$ & $39(44)$ \\
\hline Medical story of difficult intubation, $n$ (\%) & $4 / 59(7)$ & $0 / 54(0)$ \\
\hline Mouth opening less than $3 \mathrm{~cm}, n / n$ tot $^{\mathrm{b}}(\%)$ & $8 / 71(11)$ & $11 / 78(15)$ \\
\hline Limitation of cervical mobility $\leq 35^{\circ}, n / n$ tot $^{\mathrm{b}}(\%)$ & $5 / 61(8)$ & $2 / 67(3)$ \\
\hline Mallampati III or IV, n/n tot ${ }^{\mathrm{b}}(\%)$ & $10 / 34(29)$ & $14 / 42(33)$ \\
\hline Thyroid bone to chin distance $<65 \mathrm{~mm}, n / n$ tot $^{\mathrm{b}}(\%)$ & $7 / 52(14)$ & 10/61 (16) \\
\hline MACOCHA score, mean $(S D)^{c}$ & $2.0(1.6)$ & $2.2(2.0)$ \\
\hline \multicolumn{3}{|l|}{ Urgency of intubation, $n(\%)^{d}$} \\
\hline High emergency & $16(17)$ & $16(18)$ \\
\hline Relative emergency & $66(69)$ & $59(66)$ \\
\hline Deferred emergency & $13(14)$ & $14(16)$ \\
\hline \multicolumn{3}{|l|}{ Preoxygenation, $n(\%)$} \\
\hline 4 min or more & $90(97)$ & $81(91)$ \\
\hline Leaks around the device $^{e}$ & $5(5)$ & $11(12)$ \\
\hline Preventive fluid loading before induction & $41(43)$ & $37(42)$ \\
\hline \multicolumn{3}{|l|}{ Intubation } \\
\hline First operator, junior, $n(\%)^{f}$ & $80(84)$ & $75(84)$ \\
\hline \multicolumn{3}{|l|}{ Drugs for rapid sequence induction } \\
\hline \multicolumn{3}{|l|}{ Hypnotic agent, $n(\%)$} \\
\hline Propofol & $17(18)$ & $17(19)$ \\
\hline Etomidate & $62(65)$ & $57(65)$ \\
\hline Ketamine & $12(13)$ & $11(12)$ \\
\hline Other & $3(3)$ & $4(4)$ \\
\hline None & $1(1)$ & 0 \\
\hline \multicolumn{3}{|l|}{ Neuromuscular blocking agent, $n(\%)^{9}$} \\
\hline Succinylcholine & $64(67)$ & $57(65)$ \\
\hline Rocuronium & $23(25)$ & $22(25)$ \\
\hline Other & $3(3)$ & $4(5)$ \\
\hline None & $5(5)$ & $6(7)$ \\
\hline
\end{tabular}

$B M I$ body mass index, SAPSII simplified acute physiological score: used to assess the severity of illness: range 0-194, with higher scores indicating higher risk of death, COPD chronic obstructive pulmonary disease, HFNC high-flow therapy by nasal cannulae

a Calculated as weight in kilograms divided by height in square meters

b COPD was considered if obstructive syndrome had been documented on functional explorations

c McCabe classification: cat 1, nonfatal disease; cat 2, ultimately fatal disease (within 4 years); cat 3, rapidly fatal disease

d KNAUS chronic health status: class A, normal health status; class B, moderate activity limitation; class C, severe activity limitation due to chronic disease; class D, bedridden patient

e Leaks around the device were defined as leaks around the face mask during inspiration or expiration (SMO) or leaks by the mouth when breathing with the mouth open (HFNC)

${ }^{f}$ Residents were considered as junior operators. Doctors of medicine were considered as seniors

$\mathrm{g}$ Some patients received several drugs

$0.38,95 \%$ CI $0.15-0.95, P=0.03)$. No patient in the HFNC group presented moderate complications vs. $7 \%$ in the SMO group, $P=0.01$ (Table 3 and see Supplement 3). Multivariable analysis showed that HFNC was associated with less desaturation $<90 \%$, less intubationrelated complications, and a trend to reduce oxygen desaturation $<80 \%(P=0.058)$ (see Table 4 and Supplement 3 ). When the complications during intubation and the following hour were pooled, the results followed the same trends (see Supplement 3). The description of the drugs used for RSI and the type of adverse events are described in Supplement 3. 
Table 3 Primary and secondary outcomes

\begin{tabular}{|c|c|c|c|c|}
\hline & $\begin{array}{l}\text { High-flow nasal } \\
\text { cannulae } \\
n=95\end{array}$ & $\begin{array}{l}\text { Standard bag-valve mask } \\
\text { oxygenation } \\
n=89\end{array}$ & $P$ & $\begin{array}{l}\text { Relative risk or difference } \\
(95 \% \mathrm{CI})\end{array}$ \\
\hline \multicolumn{5}{|l|}{ Primary outcome } \\
\hline Lowest $\mathrm{SpO}_{2}$, median [IQR], \%, ITT analysis & $100[97 ; 100]$ & $99[95 ; 100]$ & 0.30 & \\
\hline Lowest $\mathrm{SpO}_{2}, \min -\max , \%$ & $69-100$ & $43-100$ & & \\
\hline \multicolumn{5}{|l|}{ Secondary outcomes } \\
\hline \multicolumn{5}{|l|}{ Preoxygenation } \\
\hline $\mathrm{SpO}_{2}$ at the beginning, median $[\mathrm{IQR}], \%$ & $98[96 ; 100]$ & $98[97 ; 100]$ & 0.75 & \\
\hline $\mathrm{SpO}_{2}$ at the end, median [IQR], $\%$ & $100[100 ; 100]$ & $100[100 ; 100]$ & 0.66 & \\
\hline Jaw-thrust maneuver, $n(\%)^{\mathrm{a}}$ & $12 / 94(13)$ & $29(33)$ & 0.0009 & $0.38(0.21-0.71)$ \\
\hline Failure to reach $\mathrm{SpO}_{2} 90 \%, n(\%)$ & $2(2)$ & 0 & 0.19 & \\
\hline \multicolumn{5}{|l|}{ Intubation } \\
\hline Two or more operators, $n(\%)$ & $27(28)$ & $14(16)$ & 0.06 & $1.74(0.97-3.12)$ \\
\hline Length, median $[\mathrm{IQR}]$, mins ${ }^{b}$ & $1[0.5 ; 1.9]$ & $0.8[0.5 ; 1.4]$ & 0.04 & \\
\hline Successful intubation, $n(\%)$ & $95(100)$ & $89(100)$ & 0.9 & \\
\hline Difficult intubation, $n(\%)^{c}$ & $9(10)$ & $1(1)$ & 0.01 & $8.58(1.04-70.82)$ \\
\hline IDS score, median [IQR $]^{d}$ & $3[2 ; 5]$ & $3[2 ; 4]$ & 0.10 & \\
\hline Mask ventilation for $\mathrm{SpO}_{2}<90 \%, n(\%)$ & $1(1)$ & $5(6)$ & 0.08 & $0.16(0.02-1.62)$ \\
\hline $\mathrm{SpO}_{2}<95 \%, n(\%)$ & $11(12)$ & $20(23)$ & 0.045 & $0.51(0.26-0.99)$ \\
\hline $\mathrm{SpO}_{2}<90 \%, n(\%)$ & $6(6)$ & $12(14)$ & 0.1 & $1.09(0.98-1.20)$ \\
\hline \multicolumn{5}{|l|}{ Intubation-related adverse events } \\
\hline At least one complication, $n(\%)$ & $6(6)$ & $17(19)$ & 0.007 & $0.31(0.13-0.76)$ \\
\hline At least one severe complication, $n(\%)^{f}$ & $6(6)$ & $14(16)$ & 0.03 & $0.38(0.15-0.95)$ \\
\hline $\mathrm{SpO}_{2}<80 \%$ & $2(2)$ & $7(8)$ & 0.06 & $0.26(0.05-1.20)$ \\
\hline Severe hypotension ${ }^{e}$ & $4(4)$ & $8(9)$ & 0.18 & $0.45(0.13-1.48)$ \\
\hline Cardiac arrest & $1(1)$ & 0 & 0.36 & - \\
\hline At least one moderate complication, $n(\%)^{f}$ & 0 & $6(7)$ & 0.01 & - \\
\hline Esophageal intubation & 0 & $5(6)$ & 0.02 & - \\
\hline Aspiration & 0 & $2(2)$ & 0.15 & - \\
\hline \multicolumn{5}{|l|}{ Morbidity in the ICU after intubation } \\
\hline Time on ventilator, median [IQR], days & $3[2 ; 6]$ & $3[2 ; 7]$ & 0.80 & \\
\hline Ventilator-associated pneumonia, $n$ (\%) & $8(8)$ & $10(11)$ & 0.55 & $0.77(0.32-1.84)$ \\
\hline SOFA score from day $1-5$, median [IQR] & $5[3 ; 9]$ & $6[4 ; 10]$ & 0.26 & \\
\hline Length of stay in ICU, median [IQR], days & $5[3 ; 12]$ & $6[3 ; 11]$ & 0.89 & \\
\hline Death, $n(\%)$ & $25(26)$ & $23(26)$ & 0.90 & $1.03(0.65-1.65)$ \\
\hline Mortality at day $28, n(\%)$ & $26(27)$ & $24(27)$ & 0.93 & $0.99(0.90-1.24)$ \\
\hline
\end{tabular}

Bold values represent $P<0.05$

SOFA sequential organ failure assessment used to assess the degree of dysfunction of five organ systems: respiratory, cardiovascular, renal, neurologic, hepatic. Each subscore ranged from 0 (healthy) to 4 (maximum severity); the overall score ranged from 0 to 20 [33]

In the HFNC group, one patient experienced a cardiac arrest secondary to deep desaturation during intubation because of difficult airway control (body mass index $>45$ )

ICU intensive care unit, $\mathrm{SpO}_{2}$ arterial saturation measured by pulse oximetry, $\mathrm{PaO}_{2}$ partial pressure of oxygen in arterial blood

a Defined as the need to perform subluxation to ensure airway permeability for spontaneous breathing during preoxygenation

b The length of intubation corresponds to the length of apnea. The external observer started the chronometer when the nurse injected hypnotics and stopped when the patient was connected to a ventilator

c Difficult intubation was defined as intubation duration longer than $10 \mathrm{~min}$ and/or three or more laryngoscopy attempts or use of an alternative device [20]

d IDS score was calculated after intubation to evaluate the difficulty of the intubation. IDS $<5$ means slightly difficult intubation. IDS $>5$ means moderate to major difficult intubation [21]

e Severe hypotension was defined as systolic blood pressure $<80 \mathrm{mmHg}$ or vasopressor introduction or $30 \%$ dose increment

f Some patients experienced several distinct complications 


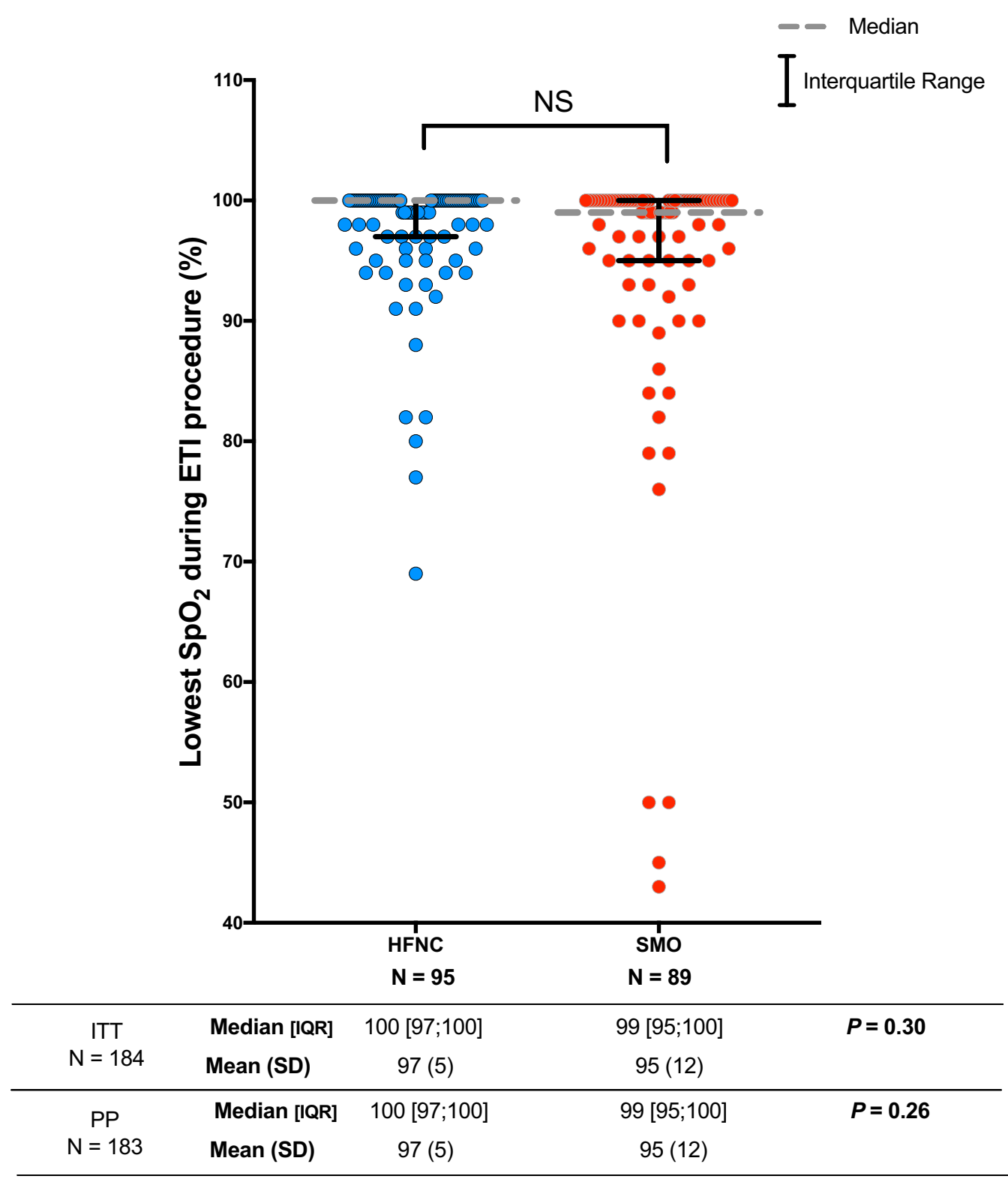

Fig. 2 Primary outcome: lowest $\mathrm{SpO}_{2}$ during the intubation procedure. HFNC high-flow nasal cannulae, SMO standard bag-valve mask oxygenation, ITT intent-to-treat analysis, PP per-protocol analysis, SD standard deviation, IQR interquartile range, $\mathrm{SpO}_{2}$ arterial oxygen saturation measured by pulse oximetry

Patients in the HFNC and SMO groups had comparable median [IQR] durations of mechanical ventilation, respectively $3[2 ; 6]$ vs. $3[2 ; 7]$ days, $P=0.80$. There was also no difference in SOFA score during the first 5 days after randomization and the length of stay in the ICU $(P=0.89)$. The mortality rate on day 28 was $27 \%$ in both groups, $P=0.93$.

\section{Discussion}

During intubation in non-severely hypoxemic critically ill patients, HFNC as a preoxygenation and apneic oxygenation device did not increase the median lowest $\mathrm{SpO}_{2}$ compared with SMO. However, HFNC significantly reduced intubation-related complications by threefold including severe complications. The latter result is a 
Table 4 Multivariable analysis for desaturation $<90 \%$ or complications during intubation

\begin{tabular}{|c|c|c|c|c|c|c|}
\hline \multirow[t]{2}{*}{$N=175^{a}$} & \multicolumn{3}{|c|}{ Desaturation $<90 \%$} & \multicolumn{3}{|c|}{ Severe and moderate complications } \\
\hline & OR & $\mathrm{Cl}$ & $P$ & OR & $\mathrm{Cl}$ & $P$ \\
\hline First operator, senior & 0.49 & $0.09-2.56$ & 0.39 & 2.07 & $0.69-6.22$ & 0.20 \\
\hline Age & 1.02 & $0.99-1.06$ & 0.22 & 1.01 & $0.98-1.04$ & 0.48 \\
\hline HFNC (vs. SMO) & 0.21 & $0.06-0.72$ & 0.01 & 0.26 & $0.08-0.77$ & 0.02 \\
\hline Difficult intubation, no & 0.08 & $0.01-0.48$ & 0.005 & 2.14 & $0.73-6.21$ & 0.16 \\
\hline MACOCHA score $\geq 3$, yes & 0.87 & $0.24-3.21$ & 0.84 & 1.00 & $1.00-1.01$ & 0.37 \\
\hline NYHA III or IV, yes & 0.32 & $0.02-4.78$ & 0.40 & 0.22 & $0.04-1.32$ & 0.10 \\
\hline $\mathrm{PaO}_{2} / \mathrm{FiO}_{2}$ at inclusion & 0.99 & $0.99-1.00$ & 0.06 & 0.93 & $0.07-12.45$ & 0.10 \\
\hline
\end{tabular}

Bold values represent $P<0.05$

In order to account for potential confounders, multivariable analysis on secondary outcomes were performed. Clinically relevant data (skill of the first operator, difficult intubation, $\mathrm{MACOCHA}$ score 3 , and $\mathrm{PaO}_{2} / \mathrm{FiO}_{2}$ ratio at baseline) or associated with the randomization group (age and NYHA class) were included in the multivariable analysis. Log linearity was checked for each parameter and adequacy of the model was verified by Hosmer and Lemeshow goodness-of-fit. Odds ratio $(\mathrm{OR})<1$ represents protective factors

a The multivariable analyses were performed in 175 patients because some data were missing for 9 patients

major finding considering the high rate of complications observed in the present study, i.e., occurring in more than $10 \%$ of patients, despite inclusion of non-severely hypoxemic patients. Considering that only one operator removed HFNC during intubation, holding the nasal prongs in place during intubation did not appear to obstruct vision or interfere with intubation.

Our team has already reported that preoxygenation in severely hypoxemic patients with HFNC compared with $\mathrm{SMO}$ did not improve median lowest $\mathrm{SpO}_{2}$ during intubation [3]. To our knowledge, the present study is the first large multicenter randomized clinical trial assessing HFNC preoxygenation before intubation in non-severely hypoxemic critically ill patients. The only publication available in this setting was the study by Miguel-Montanes et al. [15]. Their study reported an improvement in the median lowest $\mathrm{SpO}_{2}$ during intubation and a reduction in severe oxygen desaturations when using HFNC compared with face mask for preoxygenation. Although it included patients with baseline characteristics close to ours, the before-after and single-center design may have overestimated the treatment effect, limiting the generalization of the results and their reproducibility in multicenter studies. In the PROTRACH study, these trends were not confirmed, but HFNC appeared as a protective factor for desaturation $<90 \%$ in multivariable analysis (see Table 4). In line with previous studies [2, 6], and similar to hypoxemic patients [3], our results highlighted that non-severely hypoxemic patients experience numerous life-threatening adverse events during intubation including deep desaturation with minimum $\mathrm{SpO}_{2}$ of $69 \%$ in HFNC vs. $43 \%$ in SMO.

Each complication related to preoxygenation or intubation was immediately declared by the investigators and reviewed by the safety board, ensuring high reporting accuracy. Patients undergoing HFNC preoxygenation experienced fewer adverse events, including fewer severe (mainly $\mathrm{SpO}_{2}<80 \%$ ) and moderate complications. Several theoretical advantages of HFNC could explain these results. HFNC has already been reported to improve FRC, one of the main determinants of the duration of apnea without desaturation. Reducing the risk of desaturation, HFNC could in turn reduce complications such as hypotension related to hypoxemia [24]. Compared with SMO requiring two-handed thenar eminence grip to perform jaw-thrust maneuver and ensure airtightness, HFNC is a "hands-free" preoxygenation device that could help operators to be more confident and avoid scrambled intubation. Nevertheless, this aspect was not specifically assessed in the study. The reduction of desaturation $<95 \%$ in the HFNC group despite longer and more difficult intubation could also be recognized as a marker of apneic oxygenation even if this remains controversial [25]. Preoxygenation has to be differentiated from apneic oxygenation [26]. As exhaled fraction of oxygen $\left(\mathrm{EtO}_{2}\right)$ was not assessed, the reduction of desaturation in HFNC should not be interpreted as increased oxygen reserves at the end of the preoxygenation compared with SMO. However, the administration of oxygen during the apnea period with HFNC could explain the lower rate of respiratory adverse events. These results are concordant with the OPTINIV study [13]. This study showed that in severe hypoxemic patients the preoxygenation with non-invasive ventilation (NIV, namely the gold standard device in this setting $[22,27])$ combined with HFNC increased the lowest saturation during intubation compared to NIV alone. Overall, a comprehensive improvement of the intubation process including preoxygenation 
optimization, better ergonomics for the operator, and possible apneic oxygenation to offset oxygen uptake during intubation could account for these results. The technical aspect of preoxygenation with SMO can probably explain the high rate of jaw-thrust maneuver in this group.

The rate of intubation-related complications was higher in the SMO group. Strikingly, in the HFNC group difficult intubation and intubation for acute respiratory failure were more frequent, and median $\mathrm{PaO}_{2} / \mathrm{FiO}_{2}$ was lower $(P=0.009)$ and should have led to an increase in the number of desaturation and adverse events in this group. Thus, in order to account for potential baseline imbalance, a multivariable analysis was performed and found that HFNC preoxygenation was associated with a fivefold decrease in oxygen desaturation below $90 \%$ and a fourfold decrease in intubation-related complications. Nevertheless, given the small number of intubationrelated adverse events, we need to be cautious before generalizing these results.

The study includes unselected patients regardless of their admission diagnosis, in medical and surgical settings from academic and non-academic hospitals, reflecting daily practice in the ICUs and enhancing external validity. In this setting, albeit negative in terms of the primary outcome, the study provided several findings suggesting that HFNC could secure intubation by reducing complications including desaturations. Although this study enrolled ICU patients, these results could be applied to all intubations in the emergency department in non-severely hypoxemic patients considering that HFNC is now a widespread oxygenation device.

This trial has several limitations. An unblinded preoxygenation device may have interfered with our findings. Although feasible [13], blinding may have been very difficult to maintain in a large sample and multicenter trial. The choice of the primary outcome can be discussed. Considering the Miguel-Montanes et al. results, we decided to assess minimal $\mathrm{SpO}_{2}$ instead of severe desaturation below $80 \%$, a common criteria which, however, is infrequent in the non-severely hypoxemic patient. In the same way, whether a $5 \%$ difference of $\mathrm{SpO}_{2}$ is a clinically relevant endpoint may be questionable. However, such a difference can lead to a decision of bag-valve mask ventilation or can initiate a fast and deep hypoxemia given the sigmoidal relationship between the affinity of hemoglobin for oxygen and the $\mathrm{PaO}_{2}$. In this study, the 5\% difference was not met. $\mathrm{SpO}_{2}$ is not the most relevant parameter to assess oxygenation [28], but systematic arterial catheterization before intubation to measure arterial oxygen saturation or partial oxygen pressure could not be ethically defended. Similarly, $\mathrm{EtO}_{2}$ could have been a relevant way to assess the quality of preoxygenation, in particular to check that the preoxygenation was well performed in the SMO group, with a mask correctly applied to avoid leaks. It is an indicator of FRC oxygen saturation and when $\mathrm{EtO}_{2}>90 \%$ is achieved, preoxygenation can be claimed to be effective [29]. Nevertheless, in a non-occlusive device such as HFNC, measurement of $\mathrm{EtO}_{2}$ was difficult to achieve [30]. In the same way, the precise level of post-apnea end-tidal carbon dioxide $\left(\mathrm{EtCO}_{2}\right)$ was not recorded and could have provided relevant information [31]. However, we made the pragmatic choice to evaluate $\mathrm{SpO}_{2}$, the only worldwide, non-invasive, and readyto-use device informing the attending physician on apnea tolerance during intubation. Given the limitations of $\mathrm{SpO}_{2}$ measurement, a research nurse was specifically dedicated to monitor $\mathrm{SpO}_{2}$ throughout the procedure to improve data collection. Finally, NIV was not selected as the active comparator. Although it was found to improve preoxygenation in obese patients in the operating room [32], and in severe hypoxemic patients in ICU [22, 33], no data is available in non-severely hypoxemic patients in the ICU.

\section{Conclusion}

Compared with SMO, preoxygenation with HFNC provided similar lowest $\mathrm{SpO}_{2}$ during intubation in the ICU. However, HFNC was associated with enhanced safety of the procedure. These secondary results need to be confirmed and could be in favor of performing apneic oxygenation following preoxygenation in non-severely hypoxemic ICU patients.

Electronic supplementary material

The online version of this article (https://doi.org/10.1007/s00134-019-05529 -w) contains supplementary material, which is available to authorized users.

\begin{abstract}
Author details
1 Service de Réanimation Médico Chirurgicale, USC, Centre Hospitalier du Mans, 194 Avenue Rubillard, 72037 Le Mans Cedex 9, France. ${ }^{2}$ Médecine Intensive Réanimation, INSERM CIC 1415, Centre Hospitalier Universitaire de Tours, Centre d'Etude des Pathologies Respiratoires INSERM U1100, Tours University, Tours, France. ${ }^{3}$ Plateforme de Méthodologie et de Biostatistique, Centre Hospitalier Universitaire de Nantes, Nantes, France. ${ }^{4}$ Médecine Intensive Réanimation, Centre Hospitalier de La Roche sur Yon, La Roche-Sur-Yon, France. ${ }^{5}$ Médecine Intensive Réanimation, Centre Hospitalier Universitaire de Rennes, Rennes, France. ${ }^{6}$ Médecine Intensive Réanimation, Centre Hospitalier Universitaire de Brest, Brest, France. ${ }^{7}$ Service d'Anesthésie-Réanimation Chirurgicale, Hôtel-Dieu, Centre Hospitalier Universitaire de Nantes, Nantes, France.

${ }^{8}$ Médecine Intensive Réanimation, Hôtel-Dieu, Centre Hospitalier Universitaire de Nantes, Nantes, France.
\end{abstract}

\section{Acknowledgements}

Collaborators and additional contributions: We are grateful to all medical staff, nurses, and research staff at the seven sites for inclusion and data collection. We thank Caroline Postnikoff (University Hospital of Nantes) for administrative and logistic support and Marion Rigot (University Hospital of Nantes) for creating the electronic Case Report Form and Dr. Anne Chiffoleau, MD for safety monitoring (University Hospital of Nantes). The safety board was composed of Dr. Christophe Girault (University Hospital of Rouen), Prof. Gilles Capellier 
(University Hospital of Besancon) and Prof. Antoine Roch (University Hospital of Marseille).

Data sharing statements: Deidentified data collected for the study, including individual participant data and a data dictionary defining each field, in the set will be made available to others. Study protocol, statistical analysis plan, informed consent form, and record of the primary outcome will be made available on reasonable request by addressing an email to the corresponding author in the 5 years following publication with investigator support.

\section{Author contributions}

CG and MV had full access to all of the data in the study and take responsibility for the integrity of the data and the accuracy of the data analysis. Study concept and design: MV, CG, OZ, CB, and SE. Data acquisition: all of the authors participated in data collection and acquisition. Data analysis and interpretation: CG, MV, CB, SE, and CV. Drafting of the manuscript: CG, MV, $\mathrm{CB}, \mathrm{OZ}$, and $\mathrm{SE}$. Critical revision of the manuscript for important intellectual content: All of the authors. Statistical analysis: CV. Funding obtained: CG and OZ. Administrative, technical, or material support: All of the clinician authors. Study supervision: MV and CG.

Financial and material support for the research and the grant support, funding source, and provision of equipment and supplies

This study was supported by the French Ministry of Health (Interregional French Clinical Hospital Research Program Grant; PHRCi 2014-API12/N/077) in addition to a grant for research \& innovation missions allocated to the University Hospital of Nantes and by Fisher \& Paykel Healthcare. The University Hospital of Nantes was the sponsor of the study. The equipment was loaned by Fisher \& Paykel Healthcare whereas nasal cannulae were bought with the funds of PHRCi.

Fisher \& Paykel participation was inferior to $5 \%$ of the total budget (3500 $€$ ). Fisher \& Paykel did not participate in the design and conduct of the study, nor in data collection, management, analysis and interpretation of the data, nor in the preparation, review, approval, and decision to submit the manuscript for publication.

\section{Compliance with ethical standards}

\section{Conflicts of interest}

Christelle Volteau, Colin Gwenaël, Adel Maamar, Vanessa Jean-Michel, Pierre Joachim Mahe, Mickaël Landais, Noëlle Brule, Cedric Bretonnière, Olivier Zambon, and Christophe Guitton declare no conflict of interest. Stephan Ehrmann declares receiving consultancy fees from Aerogen Ltd, La Diffusion Technique Française, and Baxter healthcare and unrestricted research support from Fisher and Paykel, Aerogen Ltd, and Hamilton Medical. Mickael Vourc'h declares personal fees from MSD, Pfizer, Baxter, Grants from Fisher Paykel, outside the submitted work.

\section{Ethical approval}

The appropriate ethics committee approved this study protocol in September $112015(15 / 13-975)$.

\section{Publisher's Note}

Springer Nature remains neutral with regard to jurisdictional claims in published maps and institutional affiliations.

Received: 20 November 2018 Accepted: 11 January 2019 Published online: 21 January 2019

\section{References}

1. Jaber S, Amraoui J, Lefrant J-Y et al (2006) Clinical practice and risk factors for immediate complications of endotracheal intubation in the intensive care unit: a prospective, multiple-center study. Crit Care Med 34:23552361. https://doi.org/10.1097/01.CCM.0000233879.58720.87

2. De Jong A, Rolle A, Molinari N et al (2018) Cardiac arrest and mortality related to intubation procedure in critically ill adult patients: a multicenter cohort study. Crit Care Med 46:532-539. https://doi.org/10.1097/ CCM.00000000000002925
3. Vourc'h M, Asfar P, Volteau C et al (2015) High-flow nasal cannula oxygen during endotracheal intubation in hypoxemic patients: a randomized controlled clinical trial. Intensive Care Med. https://doi.org/10.1007/s0013 4-015-3796-z

4. Jaber S, Jung B, Corne P et al (2010) An intervention to decrease complications related to endotracheal intubation in the intensive care unit: a prospective, multiple-center study. Intensive Care Med 36:248-255. https ://doi.org/10.1007/s00134-009-1717-8

5. De Jong A, Clavieras N, Conseil M et al (2013) Implementation of a combo videolaryngoscope for intubation in critically ill patients: a beforeafter comparative study. Intensive Care Med 39:2144-2152. https://doi. org/10.1007/s00134-013-3099-1

6. Lascarrou J-B, Boisrame-Helms J, Bailly A et al (2017) Video laryngoscopy vs. direct laryngoscopy on successful first-pass orotracheal intubation among ICU patients: a randomized clinical trial. JAMA 317:483-493. https //doi.org/10.1001/jama.2016.20603

7. Russotto V, Cortegiani A, Raineri SM et al (2017) Respiratory support techniques to avoid desaturation in critically ill patients requiring endotracheal intubation: a systematic review and meta-analysis. J Crit Care 41:98-106. https://doi.org/10.1016/j.jcrc.2017.05.003

8. Benumof JL (1999) Preoxygenation: best method for both efficacy and efficiency. Anesthesiology 91:603-605

9. Higgs A, McGrath BA, Goddard C et al (2018) Guidelines for the management of tracheal intubation in critically ill adults. Br J Anaesth 120:323352. https://doi.org/10.1016/j.bja.2017.10.021

10. Frat J-P, Thille AW, Mercat A et al (2015) High-flow oxygen through nasal cannula in acute hypoxemic respiratory failure. N Engl J Med 372:21852196. https://doi.org/10.1056/NEJMoa1503326

11. Roca O, Riera J, Torres F, Masclans JR (2010) High-flow oxygen therapy in acute respiratory failure. Respir Care 55:408-413

12. Vargas F, Saint-Leger M, Boyer A et al (2015) Physiologic effects of high-flow nasal cannula oxygen in critical care subjects. Respir Care 60:1369-1376. https://doi.org/10.4187/respcare.03814

13. Jaber S, Monnin M, Girard M et al (2016) Apnoeic oxygenation via highflow nasal cannula oxygen combined with non-invasive ventilation preoxygenation for intubation in hypoxaemic patients in the intensive care unit: the single-centre, blinded, randomised controlled OPTINIV trial. Intensive Care Med 42:1877-1887. https://doi.org/10.1007/s0013 4-016-4588-9

14. Badiger S, John M, Fearnley RA, Ahmad I (2015) Optimizing oxygenation and intubation conditions during awake fibre-optic intubation using a high-flow nasal oxygen-delivery system. Br J Anaesth 115:629-632. https ://doi.org/10.1093/bja/aev262

15. Miguel-Montanes R, Hajage D, Messika J et al (2015) Use of high-flow nasal cannula oxygen therapy to prevent desaturation during tracheal intubation of intensive care patients with mild-to-moderate hypoxemia. Crit Care Med 43:574-583. https://doi.org/10.1097/CCM.0000000000 000743

16. Ward JJ (2012) High-flow oxygen administration by nasal cannula for adult and perinatal patients. Respir Care 58:98-122. https://doi. org/10.4187/respcare.01941

17. Mort TC (2005) Preoxygenation in critically ill patients requiring emergency tracheal intubation. Crit Care Med 33:2672-2675

18. Frerk C, Mitchell VS, McNarry AF et al (2015) Difficult Airway Society 2015 guidelines for management of unanticipated difficult intubation in adults. Br J Anaesth 115:827-848. https://doi.org/10.1093/bja/aev371

19. Grmec S (2002) Comparison of three different methods to confirm tracheal tube placement in emergency intubation. Intensive Care Med 28:701-704. https://doi.org/10.1007/s00134-002-1290-x

20. American Society of Anesthesiologists Task Force on Management of the Difficult Airway (2003) Practice guidelines for management of the difficult airway: an updated report by the American Society of Anesthesiologists Task Force on Management of the Difficult Airway. Anesthesiology 98:1269-1277

21. Adnet F, Borron SW, Racine SX et al (1997) The intubation difficulty scale (IDS): proposal and evaluation of a new score characterizing the complexity of endotracheal intubation. Anesthesiology 87:1290-1297

22. Baillard C, Fosse J-P, Sebbane M et al (2006) Noninvasive ventilation improves preoxygenation before intubation of hypoxic patients. Am J Respir Crit Care Med 174:171-177. https://doi.org/10.1164/rccm.20050 9-15070C 
23. De Jong A, Molinari N, Terzi N et al (2013) Early identification of patients at risk for difficult intubation in the intensive care unit: development and validation of the MACOCHA score in a multicenter cohort study. Am J Respir Crit Care Med 187:832-839. https://doi.org/10.1164/rccm.20121 $0-18510 \mathrm{C}$

24. Langeron O, Bourgain J-L, Francon D et al (2017) Difficult intubation and extubation in adult anaesthesia. Anesth Réanim 3:552-571. https://doi. org/10.1016/j.anrea.2017.09.003

25. Semler MW, Janz DR, Lentz RJ et al (2015) Randomized trial of apneic oxygenation during endotracheal intubation of the critically III. Am J Respir Critical Care Med. https://doi.org/10.1164/rccm.201507-1294oc

26. Papazian L, Corley A, Hess D et al (2016) Use of high-flow nasal cannula oxygenation in ICU adults: a narrative review. Intensive Care Med. https:// doi.org/10.1007/s00134-016-4277-8

27. Quintard H, l'Her E, Pottecher J et al (2017) Intubation and extubation of the ICU patient. Anaesth Crit Care Pain Med 36:327-341. https://doi. org/10.1016/j.accpm.2017.09.001

28. Perkins GD, McAuley DF, Giles S et al (2003) Do changes in pulse oximeter oxygen saturation predict equivalent changes in arterial oxygen saturation? Crit Care 7:R67-R67. https://doi.org/10.1186/cc2339
29. Pillai A, Daga V, Lewis J et al (2016) High-flow humidified nasal oxygenation vs. standard face mask oxygenation. Anaesthesia 71:1280-1283. https://doi.org/10.1111/anae.13607

30. Ang KS, Green A, Ramaswamy KK, Frerk C (2017) Preoxygenation using the Optiflow ${ }^{\mathrm{TM}}$ system. Br J Anaesth 118:463-464. https://doi. org/10.1093/bja/aex016

31. Patel A, Nouraei SAR (2015) Transnasal humidified rapid-insufflation ventilatory exchange (THRIVE): a physiological method of increasing apnoea time in patients with difficult airways. Anaesthesia 70:323-329. https:// doi.org/10.1111/anae.12923

32. Futier E, Constantin J-M, Pelosi P et al (2011) Noninvasive ventilation and alveolar recruitment maneuver improve respiratory function during and after intubation of morbidly obese patients: a randomized controlled study. Anesthesiology 114:1354-1363. https://doi.org/10.1097/ ALN.0b013e31821811ba

33. Baillard C, Prat G, Jung B et al (2018) Effect of preoxygenation using noninvasive ventilation before intubation on subsequent organ failures in hypoxaemic patients: a randomised clinical trial. Br J Anaesth 120:361367. https://doi.org/10.1016/j.bja.2017.11.067 